\title{
LRG1 is an adipokine that mediates obesity-induced hepatosteatosis and insulin resistance
}

\author{
Sijia He, ${ }^{1}$ Jiyoon Ryu, ${ }^{2}$ Juanhong Liu, ${ }^{3}$ Hairong Luo, ${ }^{3}$ Ying Lv, ${ }^{4}$ Paul R. Langlais, ${ }^{5}$ Jie Wen, ${ }^{3}$ Feng Dong, ${ }^{6}$ Zhe Sun, ${ }^{4}$ Wenjuan Xia, ${ }^{4}$ \\ Jane L. Lynch, ${ }^{7}$ Ravindranath Duggirala, ${ }^{8}$ Bruce J. Nicholson, ${ }^{6}$ Mengwei Zang, ${ }^{9}$ Yuguang Shi, ${ }^{1}$ Fang Zhang, ${ }^{4}$ Feng Liu, ${ }^{3}$ Juli Bai, ${ }^{1,3}$ \\ and Lily Q. Dong²

\begin{abstract}
'Department of Pharmacology and 'Department of Cell Systems \& Anatomy, University of Texas Health at San Antonio, San Antonio, Texas, USA. ${ }^{3}$ National Clinical Research Center for Metabolic Diseases, Metabolic Syndrome Research Center, Key Laboratory of Diabetes Immunology, Ministry of Education, and Department of Metabolism and Endocrinology, The Second Xiangya Hospital of Central South University, Changsha, Hunan, China. ${ }^{4}$ Novo Nordisk Research Centre China, Beijing, China. ${ }^{5}$ Department of Medicine, University of Arizona, Tucson, Arizona, USA. ${ }^{6}$ Department of Biochemistry and Structural Biology, University of Texas Health at San Antonio, San Antonio, Texas, USA. 'Department of Pediatrics, University of Texas Health at San Antonio, San Antonio, Texas, USA. ${ }^{8}$ Department of Human Genetics and South Texas Diabetes and Obesity Institute, School of Medicine, University of Texas Rio Grande Valley, McAllen, Texas, USA. ${ }^{9}$ Department of Molecular Medicine, University of Texas Health at San
\end{abstract} \\ Antonio, San Antonio, Texas, USA.
}

\begin{abstract}
Dysregulation in adipokine biosynthesis and function contributes to obesity-induced metabolic diseases. However, the identities and functions of many of the obesity-induced secretory molecules remain unknown. Here, we report the identification of leucine-rich alpha-2-glycoprotein 1 (LRG1) as an obesity-associated adipokine that exacerbates high fat diet-induced hepatosteatosis and insulin resistance. Serum levels of LRG1 were markedly elevated in obese humans and mice compared with their respective controls. LRG1 deficiency in mice greatly alleviated diet-induced hepatosteatosis, obesity, and insulin resistance. Mechanistically, LRG1 bound with high selectivity to the liver and promoted hepatosteatosis by increasing de novo lipogenesis and suppressing fatty acid $\beta$-oxidation. LRG1 also inhibited hepatic insulin signaling by downregulating insulin receptor substrates 1 and 2 . Our study identified LRG1 as a key molecule that mediates the crosstalk between adipocytes and hepatocytes in diet-induced hepatosteatosis and insulin resistance. Suppressing LRG1 expression and function may be a promising strategy for the treatment of obesity-related metabolic diseases.
\end{abstract}

\section{Introduction}

Adipose tissue dysfunction plays a critical role in the development of insulin resistance (IR), a major risk factor for type 2 diabetes, fatty liver diseases, and cardiovascular complication $(1,2)$. Aside from functioning as a major energy storage organ, adipose tissue has been recognized as an endocrine organ that mediates many biological processes (3), including glucose metabolism (4), inflammation (5), and angiogenesis (6). A number of secretory molecules such as leptin (7), adiponectin (8), and retinol binding protein 4 (RBP4) (9) have been identified in adipocytes, and dysregulated expression, secretion, and function of these adipokines are associated with obesity, IR, and cardiovascular complications (10). However, the identities and functions of many other adipokines in obesity-related metabolic diseases remain largely unclear.

Leucine-rich alpha-2-glycoprotein 1 (LRG1), which was initially isolated from human plasma (11), is a member of a highly conserved protein family that contains the leucine-rich-repeat (LRR) domains (12). In addition to regulating angiogenesis (12), LRG1 has also been implicated in a number of diseases such as cancer (13-15), arterial stiffness (16), heart failure (17), aging (18), and

Conflict of interest: The authors have declared that no conflict of interest exists. Copyright: (5) 2021, American Society for Clinical Investigation.

Submitted: February 9, 2021; Accepted: October 27, 2021; Published: December 15, 2021. Reference information: J Clin Invest. 2021;131(24):e148545.

https://doi.org/10.1172/JCl148545. inflammatory disorders (19). However, the function and mechanisms of action of LRG1 in metabolism remain unknown.

Here, we identify LRG1 as an obesity-induced adipokine that exacerbates diet-induced metabolic dysfunction. LRG1 binds with high selectivity to mouse liver and mediates obesityinduced hepatosteatosis. LRG1 also suppresses insulin signaling in hepatocytes by downregulating insulin receptor substrate 1 and substrate 2 (IRS1 and IRS2) expression. Our study reveals LRG1 as a potential target for therapeutic treatment of obesityassociated metabolic diseases.

\section{Results}

Identification of LRG1 as an adipokine. To identify secretory molecules in fat tissues potentially involved in the regulation of energy homeostasis, we investigated gene expression profiles of mature adipocytes versus preadipocytes by microarray expression analysis (Supplemental Figure 1A; supplemental material available online with this article; https://doi.org/10.1172/JCI148545DS1). Based on the presence of potential $\mathrm{N}$-terminal signal peptides and the cellular localization of the proteins, we identified over 400 genes encoding potential secretory molecules with at least 2-fold difference using a $P$ value (false positive rate) less than or equal to 0.05 as the cutoff criteria. Among these genes, the expression levels of 134 genes were significantly altered in both brown adipocytes and 3T3-L1 white adipocytes during differentiation (Supplemental Figure 1B). From these 134 genes (Supplemental 
A

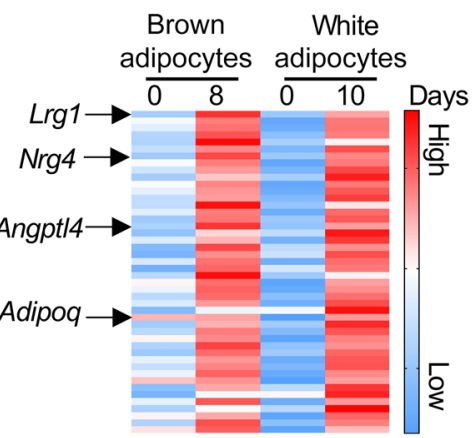

B

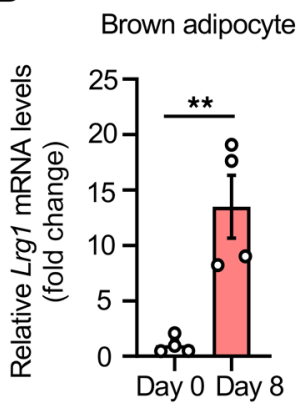

C

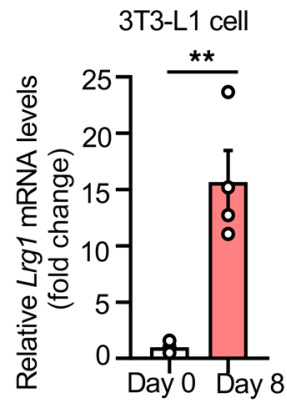

D Brown diff. 3T3-L1 diff. \begin{tabular}{llllll}
02468 & & 02468 & \\
\hline
\end{tabular}

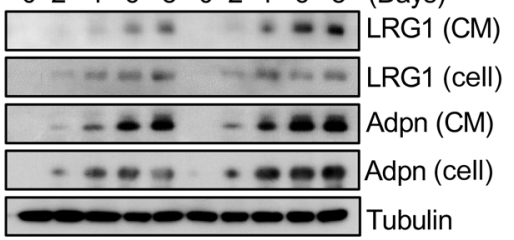

E

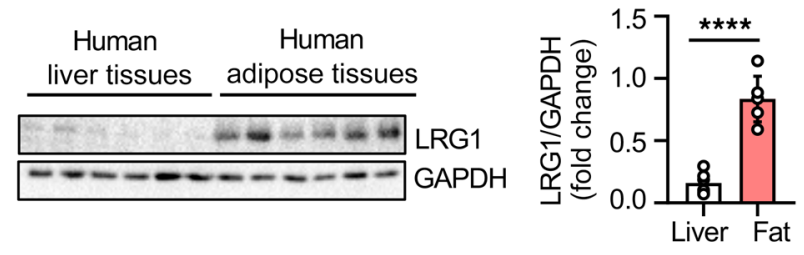

$\mathbf{F}$

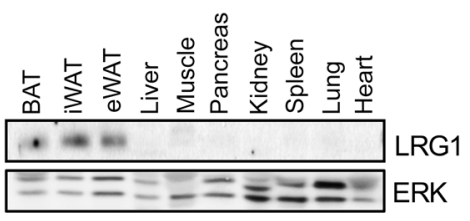

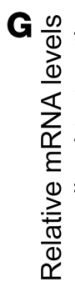

G $\frac{\omega}{d}$

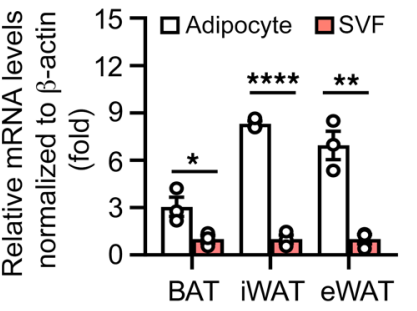

H

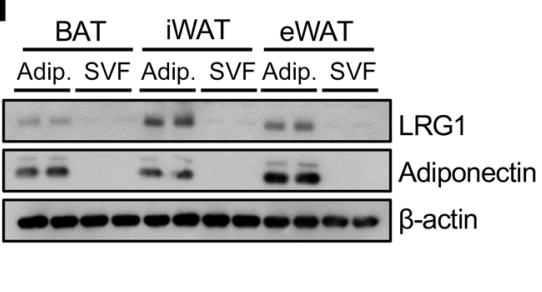

Figure 1. Identification of LRG1 as an adipokine. (A) Upregulation of secretory factors during brown and 3T3-L1 cell differentiation ( $n=3 /$ treatment group). High and low represent the value of $Z$ score. $L$ rg1 mRNA levels before and after brown (B) and white (C) adipocyte differentiation ( $n=4 /$ group). (D) LRG1 protein levels during adipocyte differentiation. CM: cell culture medium. Adpn: Adiponectin. (E) LRG1 protein levels in human liver $(n=6)$ and adipose tissues $(n=6)$. (F) Tissue distribution of LRG1 protein in 4-month-old male C57BL/6) mice after saline perfusion. (G) Lrg1 mRNA and (H) protein levels in adipocyte and SVFs of [57BL/6] mice. Adip: adipocytes. Data in $\mathbf{D}, \mathbf{F}$, and $\mathbf{H}$ are representative of 3 independent experiments. All graphical data represent mean \pm SEM. Unpaired 2-tailed $t$ test, ${ }^{*} P \leq 0.05,{ }^{* *} P \leq 0.01$, and ${ }^{* * *} P \leq 0.0001$.

Figure 1C), we identified 46 genes that were upregulated in both brown and white adipocytes (Figure 1A), including those encoding well-recognized adipokines such as adiponectin (Adipoq), neuregulin $4(\mathrm{Nrg} 4)$, and angiopoietin-like 4 (Angptl4). Interestingly, a gene named Lrg1 showed the highest levels of induction during adipocyte differentiation. Quantitative PCR (qPCR) analysis confirmed that $\operatorname{Lrg1}$ mRNA levels were significantly enhanced in fully differentiated adipocytes versus preadipocytes (Figure 1, B and C). LRG1 protein levels were also markedly induced during brown and white adipocyte differentiation, and were readily detected in the medium of cultured adipocytes (Figure 1D), indicating LRG1 as a secreted protein. While $\operatorname{Lrg} 1 \mathrm{mRNA}$ was detected in the liver (16) and other tissues in both humans (Supplemental Figure 1D) and mice (Supplemental Figure 1E), LRG1 protein expression was detected predominantly in human adipose tissue compared with liver (Figure 1E). In line with this finding, high LRG1 protein levels were almost exclusively detected in mouse fat depots including brown adipose tissue (BAT), inguinal white adipose tissue (iWAT), and epididymal white adipose tissue (eWAT; Figure $1 \mathrm{~F}$ ), but not in other tissues examined. To further dissect the source of LRG1 expression in adipose tissue, we examined LRG1 expression in adipocytes and stromal vascular fractions (SVFs) freshly purified from different fat pads. We found that both the mRNA (Figure 1G) and protein (Figure 1H) levels of LRG1 were primarily detected in adipocytes rather than SVFs, which is consistent with the finding from RNA-Seq database analysis showing that $\operatorname{Lrg} 1$ is highly enriched in adipocytes $(20,21)$. Collectively, these results demonstrate that LRG1 is an adipokine.

LRG1 levels are increased in obese mice and humans. Based on the finding that the serum levels of LRG1 correlate with diabetic complications in humans (16), we asked whether LRG1 levels are altered in obesity. The circulating levels of LRG1 were significantly elevated in obese human subjects compared with lean individuals (Figure 2A). LRG1 mRNA levels were positively correlated with body mass index (BMI) in human subcutaneous white adipose tissue (Figure 2B). Consistent with the human data, LRG1 levels were significantly higher in the serum (Figure 2C) and fat depots (Figure 2D) including BAT, iWAT, and eWAT of mice fed a high fat diet (HFD) compared with mice fed a normal chow (NC) diet. A significant increase in LRG1 protein levels was also observed in both the serum (Figure 2E) and adipose tissues (Figure 2F) of $d b / d b$ mice compared with their lean control mice. The positive correlation of LRG1 expression and secretion with obesity suggests that LRG1 may contribute to obesity-induced insulin resistance and metabolic dysfunction. Interestingly, the expression and secretion of LRG1 in adipocytes was greatly promoted by high 
A

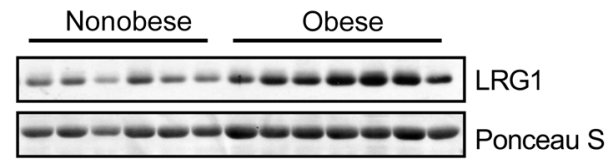

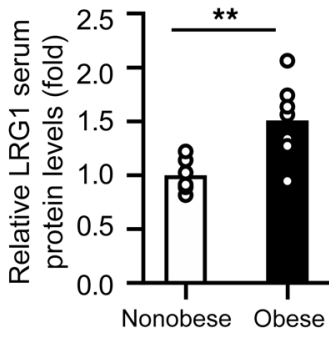

D
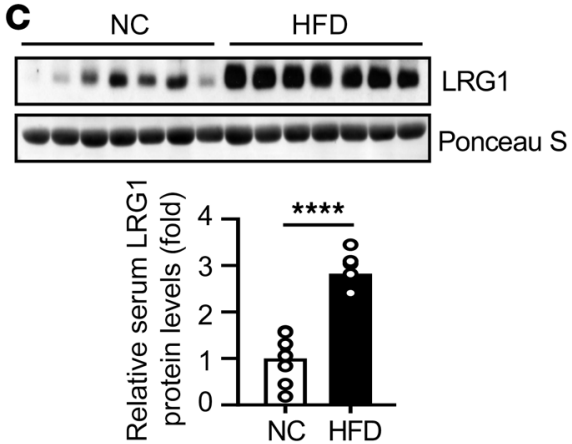

$\mathbf{F}$

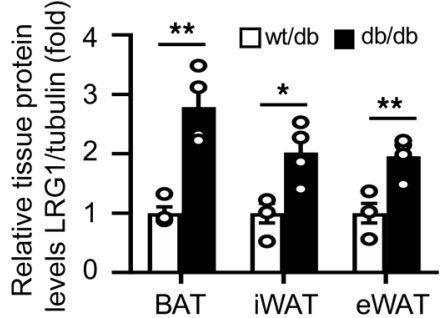

G

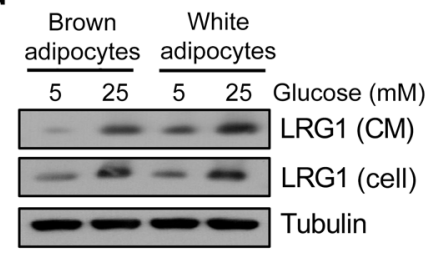

B

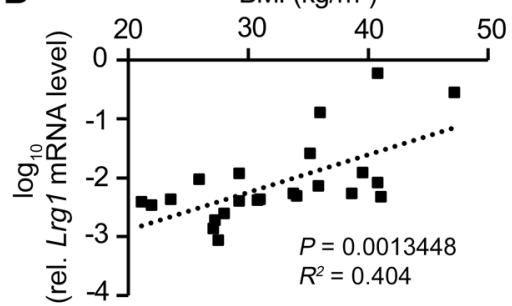

E
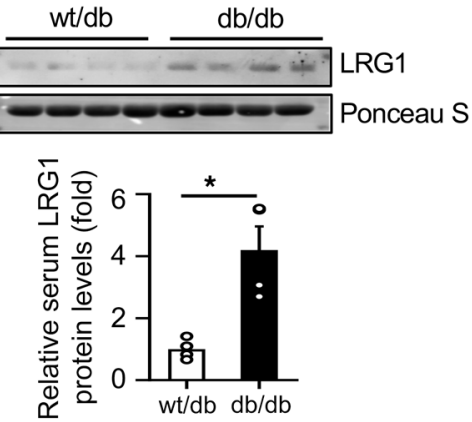

H

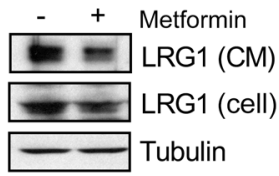

Figure 2. LRG1 is upregulated in obesity. (A) LRG1 protein levels in human serum (nonobese: $n=6$, obese: $n=7$ ). Bar graph shows quantification of the Western blot intensity using Image J software. (B) $L R G 1$ gene expression in human subcutaneous adipose tissue plotted against BMI $(n=23)$. (C) Serum LRG1 protein levels in male C57BL/6) mice fed a NC or a HFD diet ( $n=7 /$ group) for 16 weeks. (D) Quantification of LRG1 immunoblots in adipose tissues of NC- or HFD-fed C57BL/6] male mice ( $n=7 /$ group). (E) LRG1 protein levels in the serum of 4-month-old leptin receptor-deficient ( $d b / d b)$ and control mice $(w t / d b)$ ( $n=4$ /group). (F) LRG1 protein levels in adipose tissues of $d b / d b$ and $w t / d b$ mice ( $n=4$ per group). (G) LRG1 protein levels in cells and cell culture medium (CM) after glucose treatment for 48 hours. (H) LRG1 protein levels in cells and cell culture medium (CM) after 1 mM metformin treatment for 48 hours. Data in $\mathbf{G}$ and $\mathbf{H}$ are representative of $\mathbf{3}$ independent experiments. Data in $\mathbf{B}$ were analyzed using linear regression. The rest of the graphical data represent mean \pm SEM. Unpaired 2-tailed $t$ test, ${ }^{*} P \leq 0.05,{ }^{* *} P \leq 0.01,{ }^{* *} P \leq 0.001,{ }^{* * * *} P \leq 0.0001$.

glucose stimulation (Figure 2G). On the other hand, metformin treatment markedly suppressed LRG1 expression and secretion in adipocytes (Figure $2 \mathrm{H}$ ).

Knockout of the Lrg1 gene protects mice from diet-induced obesity, hepatosteatosis, and insulin resistance. To explore the physiological role of LRG1 in metabolism, we assessed several metabolic phenotypes of $\operatorname{Lrg1}$-deficient mice $\left(\operatorname{Lrg} \mathrm{K}^{\mathrm{KO}}\right)$ and WT control mice. $\operatorname{Lrg} 1^{\mathrm{KO}}$ mice (Supplemental Figure 2, A and B) were born at a normal Mendelian ratio. Under $\mathrm{NC}$ feeding conditions, the $\operatorname{Lrg} 1^{\mathrm{KO}}$ mice showed no significant differences in body weight (Supplemental Figure 2, C-E), food intake (Supplemental Figure 2F), locomotor activity (Supplemental Figure 2, G and H), and energy expenditure (Supplemental Figure 2, I and J) compared with WT controls. Additionally, no significant difference was observed in glucose tolerance, insulin sensitivity, and fasting insulin levels between $\operatorname{Lrg}{ }^{\mathrm{KO}}$ mice and WT mice fed a NC diet (Supplemental Figure 2, K-M). Under HFD feeding conditions, however, $\operatorname{Lrg} 1^{\mathrm{KO}}$ mice showed reduced body weight gain (Figure 3A) and smaller adipocyte cell size (Figure $3, \mathrm{~B}$ and $\mathrm{C}$ ), which were correlated with a slightly increased lipolytic gene expression (Supplemental Figure 3A) and decreased lipid uptake gene expression (Supplemental Figure 3B), but without an effect on the expression of genes related to lipogenesis (Supplemental Figure 3C) and fatty acid oxidation (Supplemental Figure 3D). Of note, knockout of the Lrg1 gene had no effect on adipocyte numbers (Supplemental Figure 3, E and F) or the expression of other adipokines such as adiponectin and leptin (Supplemental Figure 3, G and H). Interestingly, despite an abundant $\operatorname{Lrg} 1 \mathrm{mRNA}$ expression in BAT, knockout of $\operatorname{Lrg} 1$ had no significant effect on the expression of thermogenic genes or uncoupling protein 1 (UCP1) levels in mice under either room temperature or cold stress conditions (Supplemental Figure 3, I-L). However, LRG1 deficiency decreased fasting insulin levels (Figure 3D), improved glucose tolerance (Figure 3E), enhanced insulin sensitivity (Figure 3F), and ameliorated hepatosteatosis (Figure 3G) in mice. Additionally, the $\operatorname{Lrg} 1^{\mathrm{KO}}$ mice exhibited increased insulin-stimulated protein kinase B (Akt) phosphorylation in the liver, fat, and skeletal muscle compared with WT controls (Figure 3, H-J). The effect of LRG1 on insulin signaling seems selective since there was no significant 
A
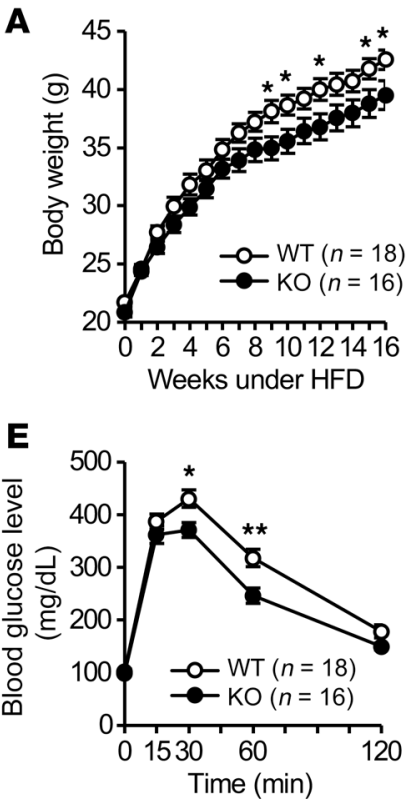

\section{H}
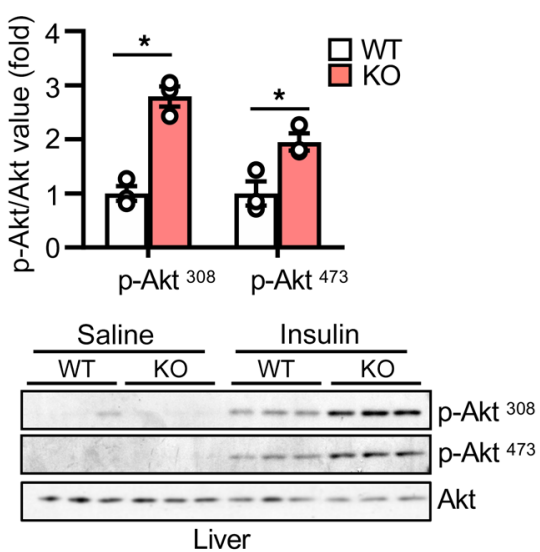
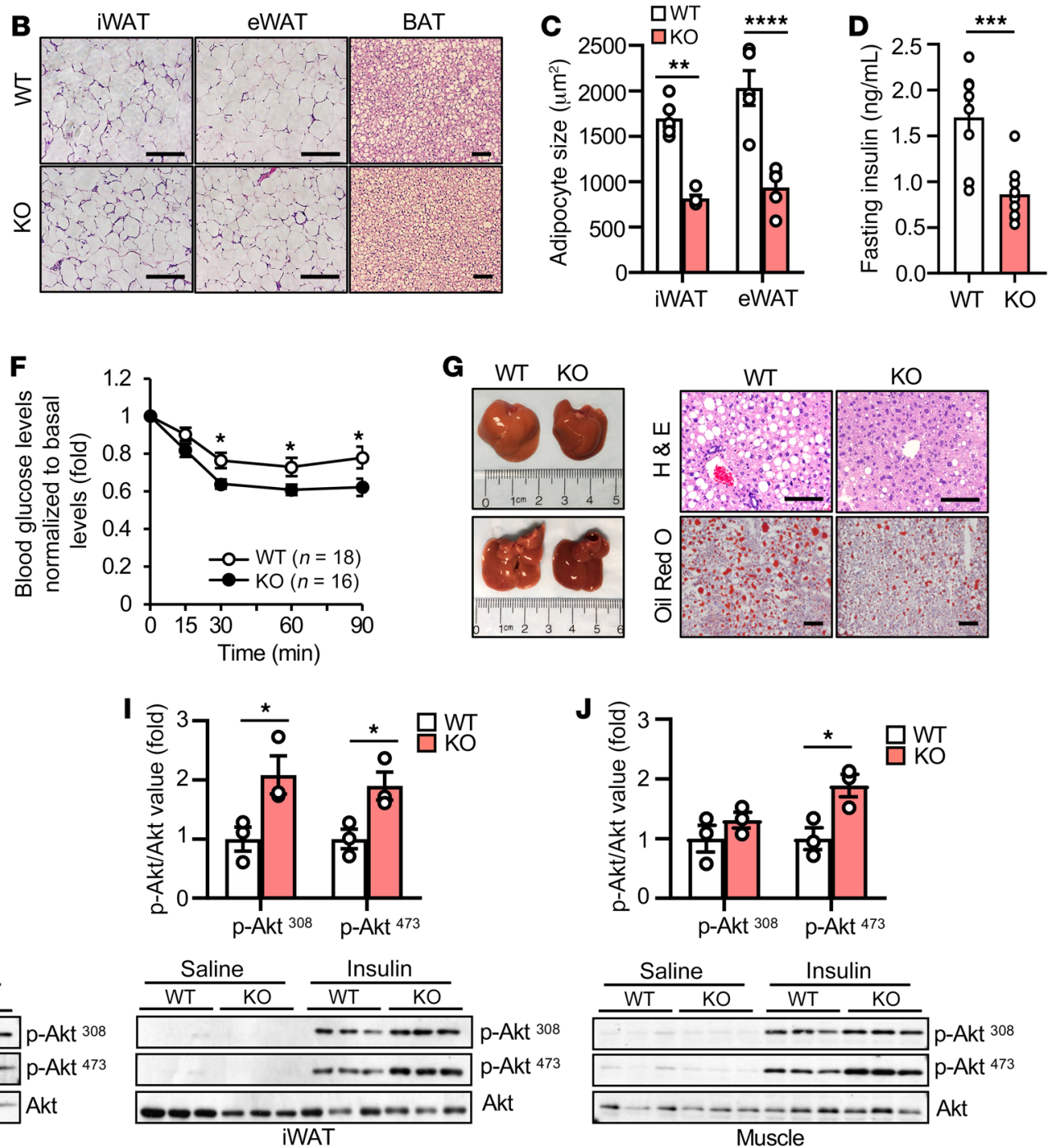

Figure 3. Lrg1 knockout protects mice from diet-induced hepatic steatosis and insulin resistance. (A) Body weight of $L$ rg $7^{\mathrm{K} 0}$ mice ( $\left.n=16\right)$ and $W T$ littermates $(n=18)$ fed a HFD for 16 weeks. (B) H\&E staining of adipose tissues from $L r g 1^{\mathrm{KO}}$ and WT mice fed with HFD for 16 weeks (scale bar: $\left.100 \mu \mathrm{m}\right)$. (C) Quantification of white adipocyte cell size based on H\&E staining ( $n=5$ sections/group). (D) Overnight fasting serum insulin levels of $L$ rg ${ }^{K 0}$ mice ( $n=9$ ) and WT littermates $(n=8)$ after 16 weeks of HFD feeding. (E) GTT and (F) ITT of $L$ rg $7^{\text {KO }}$ mice $(n=16)$ and WT littermates ( $\left.n=18\right)$ fed a HFD for 16 weeks. (G) Overall liver tissue appearance, H\&E staining, and Oil Red 0 staining of liver tissues from Lrg $7^{\mathrm{KO}}$ mice and WT littermates treated with HFD for 16 weeks (scale bar: $100 \mu \mathrm{m}$ ). (H) Liver tissue, (I) iWAT, and (J) skeletal muscle tissue was isolated from HFD-fed Lrg ${ }^{\mathrm{KO}}$ mice and control littermates injected with saline or insulin ( $n=3 /$ treatment group, $1.5 \mathrm{U} / \mathrm{kg}$ body weight, 5 minutes). Akt phosphorylation and protein levels in these tissues were determined by Western blot and quantified by Image J. Data in B and $\mathbf{G}$ are representative of 3 independent experiments. Data represent mean \pm SEM. Unpaired 2 -tailed $t$ test, ${ }^{*} P \leq 0.05,{ }^{* *} P \leq 0.01,{ }^{* * *} P \leq 0.001,{ }^{* * * *} P \leq 0.0001$.

difference in insulin-stimulated phosphorylation of the extracellular regulated MAP kinase (ERK) between HFD-fed $\operatorname{Lrg} 1^{\mathrm{KO}}$ and WT mice (Supplemental Figure 3, M-O).

Liver is the major target tissue of LRG1 action. To identify the potential target tissue(s) of LRG1 action, we generated a fusion protein with the secreted alkaline phosphatase (SEAP) fused to the N-terminus of LRG1 (SEAP-LRG1). Binding assays on frozen mouse tissue sections revealed that LRG1 binds to liver, kidney, and heart, but not to brain and skeletal muscle (Figure 4A). The binding of SEAP-LRG1 to the liver was blocked by preincubating the tissue with a competitive binding ligand LRG1 protein (LRG1Myc-His; Figure 4B), confirming the specificity of the SEAP-LRG1 binding assay. To validate the tissue-selective binding of LRG1 in vivo, we intravenously injected mice with near-infrared (NIR) fluorochrome-labeled LRG1 (Tag-LRG1). Mice were sacrificed at different time points after Tag-LRG1 injection and tissue-specific binding of Tag-LRG1 to different organs was examined ex vivo. At 16 hours after injection, substantial Tag-LRG1 fluorescence signal was detected primarily in the liver and kidney, and to a lesser extent in fat, pancreas, and bone, but not at all in the brain or skeletal muscle (Figure 4, C and D). At 48 hours after injection, binding of Tag-LRG1 was observed only in the liver (Figure 4, E and $\mathrm{F}$ ). These observations indicate that liver is one of the major target tissues for LRG1 action.

LRG1 suppresses insulin signaling and promotes gluconeogenesis in hepatocytes. To comprehensively investigate the effect of LRG1 
A
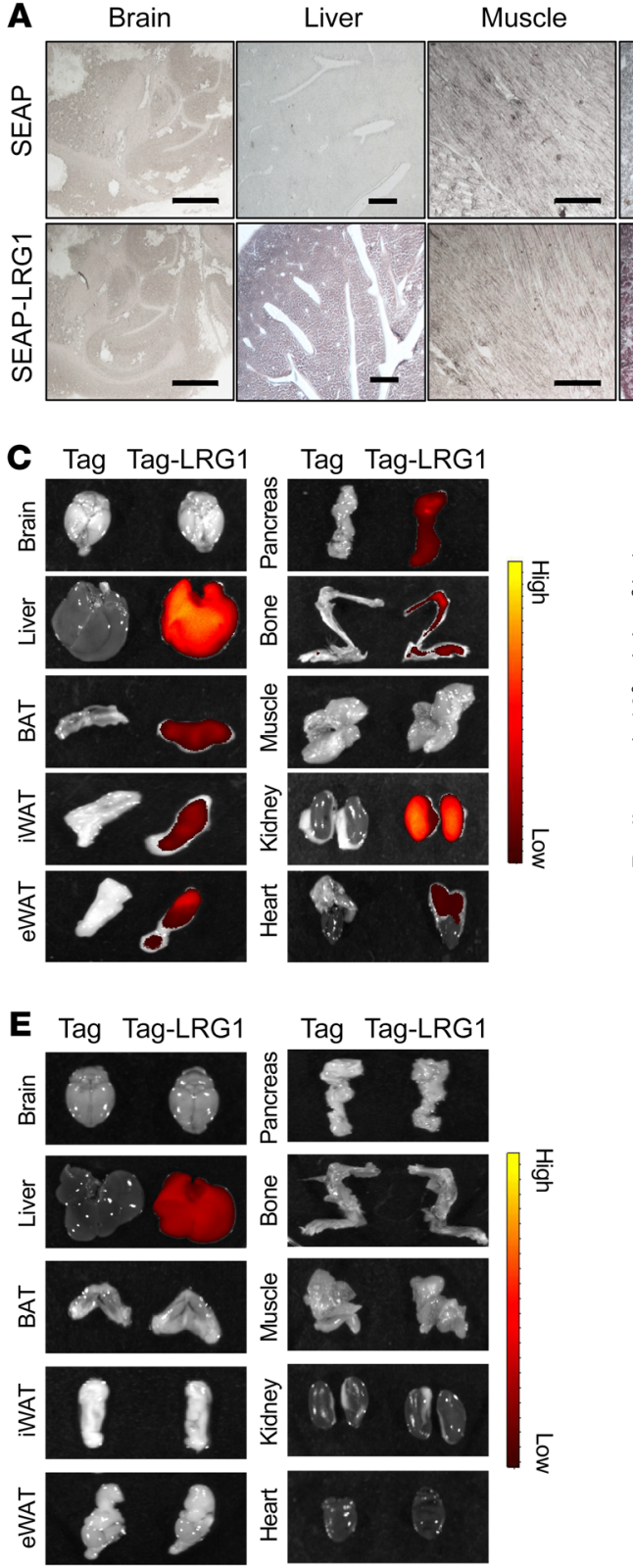

Kidney

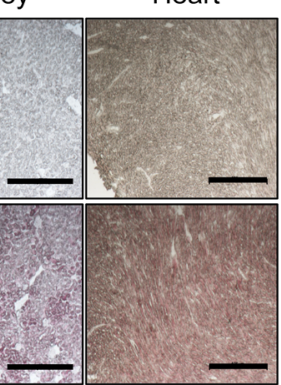

B

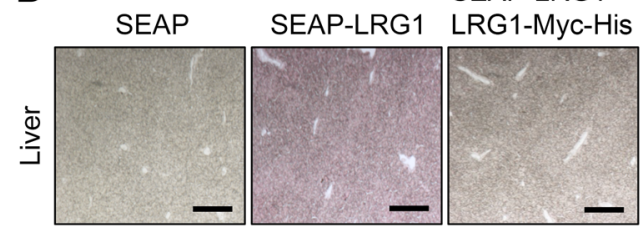

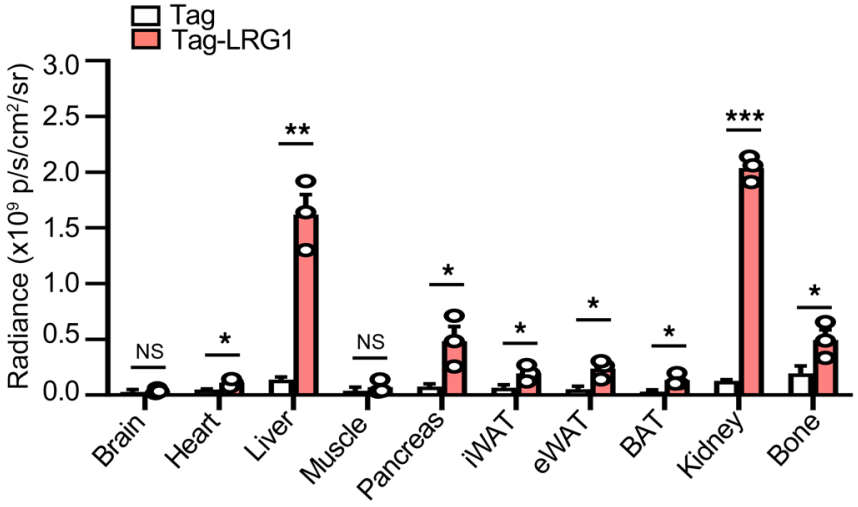

$\mathbf{F}$

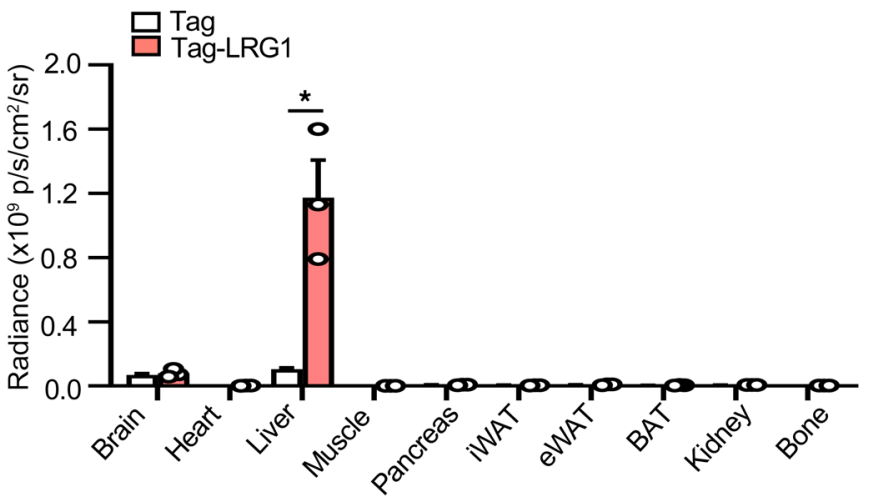

Figure 4. Identification of liver as a major target tissue of LRG1. (A) Binding of SEAP or SEAP-LRG1 to frozen tissue sections prepared from male C57BL/6) mice (scale bar: $1000 \mu \mathrm{m}$ for brain and liver, $500 \mu \mathrm{m}$ for muscle, kidney, and heart). (B) Binding of SEAP-LRG1 to liver tissue with or without preincubation of purified Myc-His-tagged recombinant LRG1 (scale bar: $1000 \mu \mathrm{m}$ ). (C) Biodistribution of LRG1 in vivo 16 hours after i.v. injection. Organs isolated from mice injected with Vivo tag680 (Tag) or Vivo Tag680-LRG1(Tag-LRG1) were subjected to Epi-luminescence imaging ( $n=3 /$ group). The color bar indicates the intensity of florescence signal based on radiance values (photons/second/ $\mathrm{cm}^{2} / \mathrm{steradian).} \mathrm{(D)} \mathrm{Quantification} \mathrm{of} \mathrm{LRG1} \mathrm{in} \mathrm{vivo} \mathrm{biodistribution}$ 16 hours after i.v. injection, data were calculated based on radiance values of each tissue (photons/second/cm²/steradian). (E) Epi-luminescence imaging measurement of biodistribution of LRG1 in vivo 48 hours after i.v. injection ( $n=3$ per group). (F) Quantification of LRG1 in vivo biodistribution 48 hours after i.v. injection. Data in $\mathbf{A}, \mathbf{B}, \mathbf{C}$, and $\mathbf{E}$ are representative of 3 independent experiments. Data in $\mathbf{D}$ and $\mathbf{F}$ represent mean \pm SEM. Unpaired 2 -tailed $t$ test, ${ }^{*} P \leq 0.05,{ }^{*} P \leq 0.01,{ }^{* *} P \leq 0.001$.

in the liver, we performed RNA-Seq analysis on primary hepatocytes treated with or without LRG1. Among several gene clusters identified (Supplemental Figure 4A), genes involved in insulin response (Supplemental Figure 4B) and lipid metabolism (Supplemental Figure 4C) were greatly altered by LRG1 treatment. Consistently, a separate RNA-Seq analysis on liver tissues of HFD-fed WT and $\operatorname{Lrg} 1^{\mathrm{KO}}$ mice revealed that many genes involved in lipid, glucose, and drug metabolism were greatly altered (Sup- plemental Figure 4, D and E). It is interesting to note that while LRG1 has been shown to be involved in pathogenesis of inflammation in rheumatoid arthritis and inflammatory bowel disease (22), knockout of Lrg1 had no significant effect on the expression of inflammatory and hepatokine genes in the liver of HFD-fed mice (Supplemental Figure 4, F and G).

To determine whether LRG1 has a direct effect on hepatic insulin signaling, we treated mouse primary hepatocytes with 
A

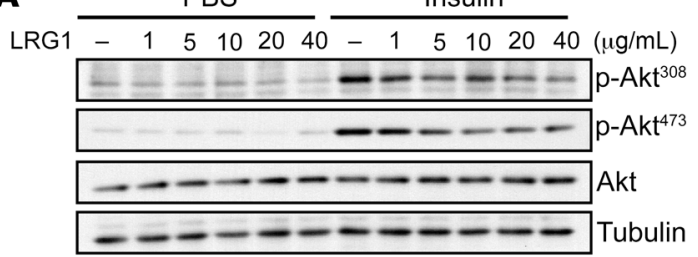

C

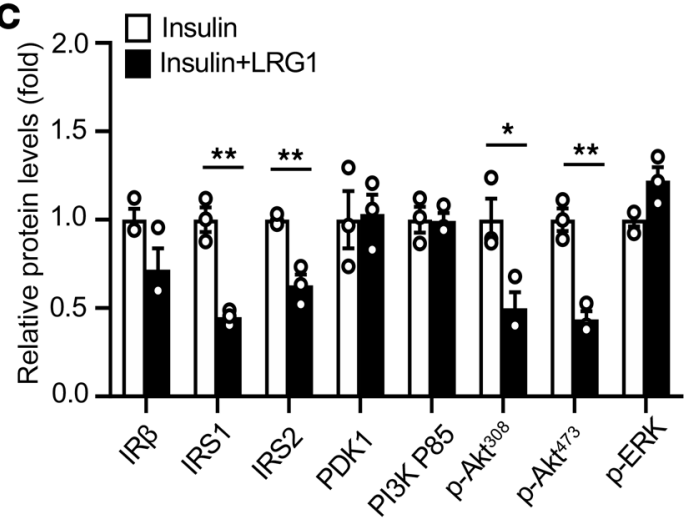

B

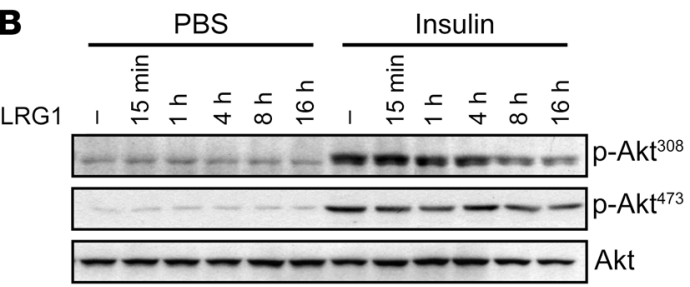

D
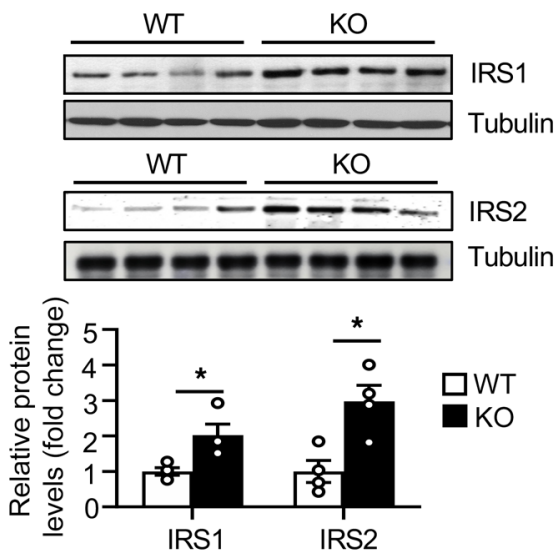

E

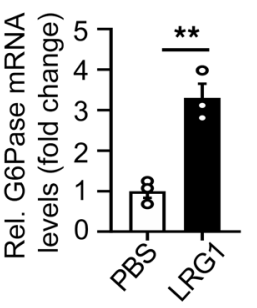

$\mathbf{F}$

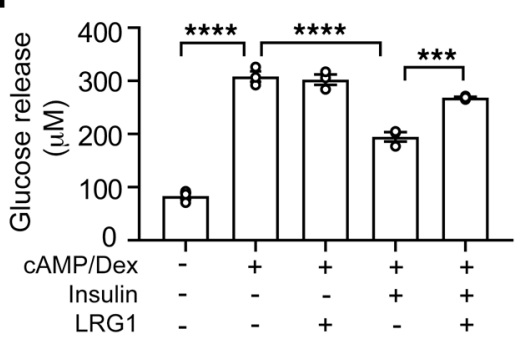

Figure 5. LRG1 promotes insulin resistance through downregulation of IRS expression in hepatocytes. (A) Dosage effect of LRG1 protein treatment on insulin signaling in hepatocytes. Primary hepatocytes from [57BL/6] mice were pretreated with different doses of LRG1 for 16 hours before treated with $10 \mathrm{nM}$ insulin for 5 minutes. (B) Time effect of LRG1 protein treatment on insulin signaling in mouse primary hepatocytes. Cells were pretreated with LRG1 at $20 \mu \mathrm{g} / \mathrm{mL}$ for indicated lengths of time prior to stimulation with $10 \mathrm{nM}$ insulin for 5 minutes. (C) Protein and/or its phosphorylation levels of insulin signaling components in primary hepatocytes treated with or without LRG1 ( $20 \mu \mathrm{g} / \mathrm{mL}, 16$ hours) prior exposure to insulin (10 $\mathrm{nM}, 5$ minutes) ( $n=3 /$ treatment group). (D) IRS1/2 protein levels in the liver tissue of WT and $L \mathrm{rg} 1^{\mathrm{K} 0}$ mice after fed with HFD for 16 weeks ( $n=4 / \mathrm{group}$ ). (E) qPCR evaluation of G6Pase mRNA levels in hepatocytes treated with or without LRG1 $(20 \mu \mathrm{g} / \mathrm{mL})$ for 1 hour ( $n=3 /$ group). (F) The effect of LRG1 (20 $\mu \mathrm{g} /$ $\mathrm{mL}, 16$ hours) on insulin-induced suppression of gluconeogenesis in mouse primary hepatocytes ( $n=3 /$ treatment group). Primary hepatocytes from C57BL/6] mice were treated with the reagents as indicated, the glucose release was then measured by colorimetric method. All cell experiments were independently repeated for 3 times. Data represent mean \pm SEM. Unpaired 2-tailed $t$ test for (C-E). One-way ANOVA followed by Tukey's test for $\mathbf{F}$. ${ }^{*} P \leq 0.05,{ }^{* *} P \leq 0.01,{ }^{* * *} P \leq 0.001,{ }^{* * *} P \leq 0.0001$.

LRG1. Insulin-stimulated phosphorylation of Akt was inhibited by LRG1 in a dose- and time-dependent manner (Figure 5, A and B). However, LRG1 treatment had no effect on ERK phosphorylation (Figure 5C and Supplemental Figure 5A), suggesting that the PI3K-Akt pathway is the primary LRG1 target downstream of the insulin receptor. Furthermore, LRG1 treatment suppressed the expression of insulin receptor substrate IRS1 and IRS2, but had no effect on the protein levels of insulin receptor $\beta$ subunit (IR- $\beta$ ), PI3K-p85, 3-phosphoinositide-dependent protein kinase-1 (PDK1) or Akt (Figure 5C and Supplemental Figure 5A). Consistent with these results, the protein levels of IRS1 and IRS2 were upregulated in the liver of $\operatorname{Lrg} 1^{\mathrm{KO}}$ mice compared with the control littermates fed with HFD (Figure 5D), suggesting a possible mechanism underlying the increased insulin signaling in LRG1-deficient mice. Treating primary hepatocytes with
LRG1 also induced the expression of the gluconeogenic gene glucose-6-phosphatase (G6Pase; Figure 5E), and attenuated the suppressive effect of insulin on gluconeogenesis (Figure 5F), further suggesting an inhibitory effect of LRG1 on hepatic insulin signaling. It is interesting to note that LRG1 treatment had no significant effect on insulin-stimulated Akt or ERK phosphorylation in both brown and 3T3-L1 adipocytes as well as mouse primary adipocytes (Supplemental Figure 5, B-D), indicating that adipocytes are not the primary target of LRG1 action. Given that LRG1 has been shown to modulate the transforming growth factor beta (TGF- $\beta$ ) signaling pathway in endothelial cells (12), we tested whether TGF- $\beta$ signaling is involved in LRG1-mediated inhibition of insulin signaling in hepatocytes. Knockout of transforming growth factor beta receptor $2(\mathrm{Tg} f b r 2)$ in primary hepatocytes (Supplemental Figure 5E) or suppressing TGF- $\beta$ 

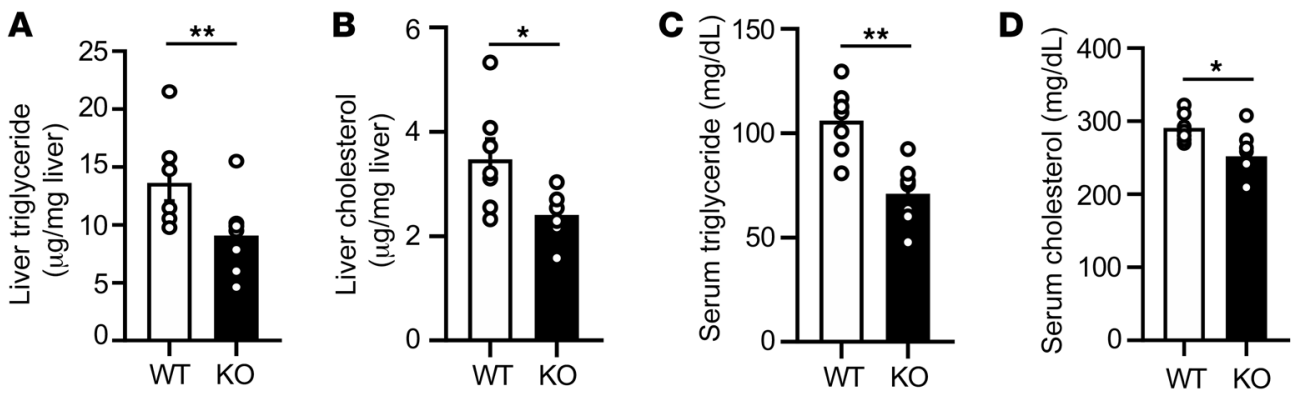

$\mathbf{E}$

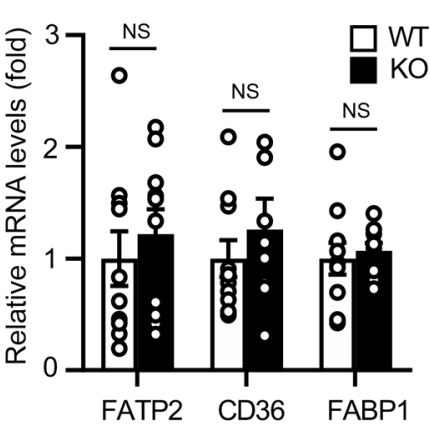

H
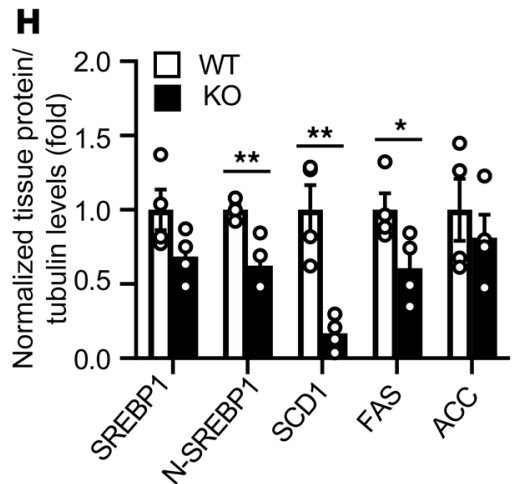

I

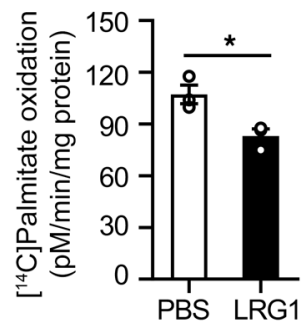

$\mathbf{F}$

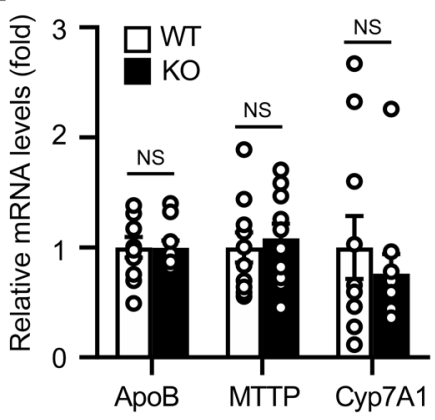

G

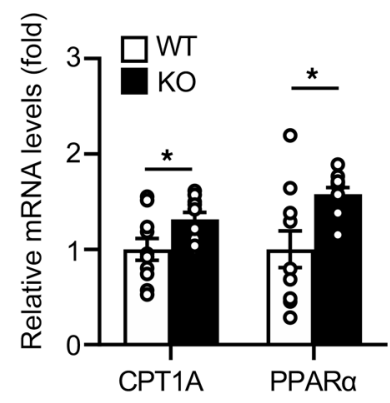

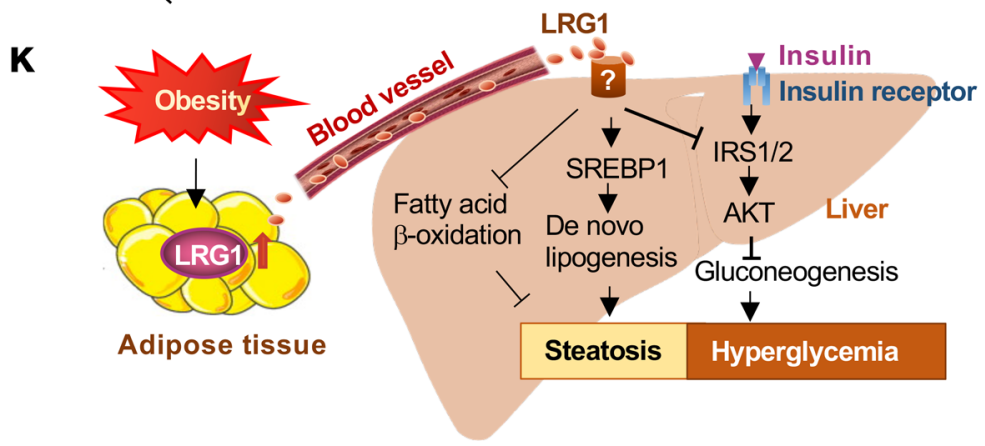

Figure 6. LRG1 contributes to diet-induced hepatic steatosis through suppressing $\beta$-oxidation and promoting de novo lipogenesis. Lrg $7^{\mathrm{K} 0}$ mice and WT control mice were under HFD feeding for 16 weeks. (A) Liver triglyceride content, (B) liver cholesterol levels, (C) serum triglyceride contents, and (D) serum cholesterol levels of these mice were detected ( $n=7$ mice/group). qPCR determination of the expression of genes involved in lipid uptake (E), lipid export $(\mathbf{F})$, and fatty acid $\beta$-oxidation (C) in the liver tissues of $L r g 7^{\mathrm{KO}}$ and WT littermates fed a HFD for 16 weeks ( $n=8-10$ mice/group). (H) The relative lipogenic protein levels from the liver tissues of these mice as quantified from Western blots by Image J (4 mice/group). (I) Fatty acid $\beta$-oxidation in primary hepatocytes treated with PBS or LRG1 $(20 \mu \mathrm{g} / \mathrm{mL})$ overnight was determined by using ${ }^{14} \mathrm{C}$-labeled palmitic acid as substrate $(n=3 /$ treatment group). (J) Lipogenesis in primary hepatocytes treated with LRG1 or insulin overnight was determined by using ${ }^{14} \mathrm{C}$-labeled acetic acid as a substrate ( $n=3 /$ treatment group). All cell experiments were independently repeated for 3 times. Data represent mean \pm SEM. Unpaired 2-tailed $t$ test for (A-I). One-way ANOVA followed by Tukey's test for J. ${ }^{*} P \leq 0.05,{ }^{* *} P \leq 0.01,{ }^{* * * *} P \leq 0.0001$. (K) A proposed model on the mechanism by which LRG1 mediates obesity-induced hepatic steatosis and insulin resistance. Obesity-induced LRG1 production in adipose tissue activates SREBP1 in the liver via an endocrinal mechanism, leading to enhanced de novo lipogenesis and suppressed fatty acid $\beta$-oxidation and consequent hepatic steatosis. LRG1 also inhibits insulin signaling by suppressing IRS1/2 expression, contributing to hepatic insulin resistance and hyperglycemia. 
receptor downstream signaling component (Smad4) in hepatocellular carcinoma cells (Supplemental Figure 5F) had no effect on the inhibitory role of LRG1 in insulin signaling. These data suggest that the inhibitory effect of LRG1 on hepatic insulin signaling is independent of TGF- $\beta$ signaling.

Based on the finding that insulin signaling coordinates the metabolic response to feeding in the liver (23), we examined the potential role of LRG1 on hepatic insulin signaling under fasting/ refeeding conditions. We found that LRG1 levels were moderately increased in the circulation and WAT, but not BAT, of mice under refeeding conditions (Supplemental Figure 6, A-D). However, LRG1 deficiency had no significant effect on hepatic insulin signaling (Supplemental Figure 6E) or insulin-stimulated suppression of gluconeogenic gene expression and increase of lipid synthesis gene expression (Supplemental Figure 6, F and G) under refeeding conditions. Given that fasting and refeeding cause metabolic reprogramming that affects the expression of numerous genes or secretion of various molecules (24), it is possible that the effect of LRG1 on insulin signaling may be masked by those factors under these physiological conditions.

LRG1 suppresses fatty acid $\beta$-oxidation and promotes de novo lipogenesis in the liver. Consistent with the finding that LRG1 deficiency protected mice from HFD-induced hepatic steatosis (Figure $3 \mathrm{G}$ ), the $\operatorname{Lrg} 1^{\mathrm{KO}}$ mice showed a significant decrease in hepatic and serum triglyceride and cholesterol levels compared with control mice (Figure 6, A-D). Quantitative PCR analysis showed that there was no significant difference in the expression of genes involved in lipid uptake (Figure 6E) and export (Figure $6 \mathrm{~F}$ ) between control and $\operatorname{Lrg} 1^{\mathrm{KO}}$ mice. However, LRG1 deficiency greatly induced the expression of genes involved in fatty acid $\beta$-oxidation (Ppara and Cpt1a; Figure 6G) and significantly suppressed lipogenic gene expression as evidenced by a decrease in the protein levels of the activated nucleus form of sterol regulatory element binding transcription factor 1 (N-SREBP1), fatty acid synthase (FAS), and stearoyl-CoA desaturase-1 (SCD1; Figure 6H). Consistent with these results, LRG1 significantly suppressed fatty acid oxidation (Figure 6I) and enhanced lipogenesis (Figure 6J) in mouse primary hepatocytes. These findings reveal that LRG1 may aggravate HFD-induced hepatosteatosis by suppressing fatty acid catabolism and promoting lipid biosynthesis.

\section{Discussion}

Adipokines mediate the intra- and inter-tissue communication in our body and play important roles in maintenance of whole-body energy homeostasis. Under certain pathological situations, such as obesity, dysregulation in adipokine biosynthesis and secretion may be a critical step for the development of various metabolic disorders. Here, we report the identification and characterization of a novel adipokine, LRG1, that mediates diet-induced obesity, insulin resistance, and hepatosteatosis. LRG1 exerts its endocrine action by binding to liver with high selectivity. HFD-induced elevation of serum LRG1 exacerbates hepatosteatosis by suppressing fatty acid $\beta$-oxidation and promoting de novo lipogenesis in mice. Binding of LRG1 to liver tissues also induces hyperglycemia by inhibiting insulin signaling and promoting gluconeogenesis. Our study identifies a new mechanism that mediates a metabolic crosstalk between fat and liver in obesity, suggesting that LRG1 may be a promising drug target for therapeutic treatment of obesityinduced metabolic diseases (Figure 6K).

Lrg1 mRNA and/or LRG1 protein were detected in several cells including endothelial cells (12), granulocytes (25), and cancer cells (26), as well as in the liver $(27,28)$. Interestingly, while we detected high levels of Lrg1 mRNA in the liver, LRG1 protein was principally detected in human (Figure 1E) and mouse adipose tissues but not in liver, muscle, pancreas, kidney, and heart (Figure 1F, and Supplemental Figure 2B). The specificity of the anti-LRG1 antibody has been validated by using tissues from $\operatorname{Lrg} 1^{\mathrm{KO}}$ mice (Supplemental Figure 2B). The levels of LRG1 in serum and adipose tissue are positively associated with BMI in both humans (Figure 2, A and B) and mice (Figure 2, C-F). Together with the findings that LRG1 expression is predominantly in adipocytes but not SVFs (Figure 1, $\mathrm{G}$ and $\mathrm{H}$ ) and that the protein is secreted from adipocytes (Figure 1D), we demonstrate that adipocytes are the major cell source of LRG1 expression and secretion.

An interesting observation made in this study is that LRG1 selectively suppresses IRS expression and insulin-stimulated PI3K signaling pathway in hepatocytes. Dysregulation of IRS expression has been found in multiple obesity models $(29,30)$, which contributes to the development of insulin resistance in obese human and animals (31). However, the mechanism by which obesity promotes IRS downregulation remains largely unclear. Several transcription factors have been identified to regulate Irs1/2 gene expression. Overexpression of the peroxisome proliferator-activated receptor $\gamma$ coactivator $1 \alpha(\mathrm{Pgcla})$ increased the expression of Irs 2 but reduced the expression of Irs1 in mouse hepatocytes (32). Upregulation of Irs 2 has also been found to be promoted by forkhead box O1 (Foxo1) and phosphorylated cAMP responsive element binding protein 1 (CREB) under nutrient deprivation conditions $(33,34)$. By contrast, sterol regulatory-element binding proteins (SREBPs) suppress Irs 2 expression, at least in part by interfering with FOXO1 binding to the Irs2 promoter (33). Intriguingly, we found nuclear form N-SREBP1, but not the SREBP1 expression, markedly decreased in livers of $\operatorname{Lrg} 1^{\mathrm{KO}}$ mice, suggesting a potential mechanism by which LRG1 regulates Irs gene expression. However, whether other transcription factors are also required for both regulation of Irs1/2 expression remains to be further investigated. Nevertheless, the findings that obesity greatly upregulates LRG1 expression and that this adipokine directly targets on hepatocytes to negatively regulate IRS levels suggest a potential mechanism by which obesity suppresses hepatic insulin signaling and induces insulin resistance. Interestingly, we found that LRG1 production in adipocytes is suppressed by metformin, which has previously been shown to induce IRS expression in human granulosa cells (35). Additional studies will be needed to determine whether the insulin-sensitizing effect of metformin is mediated by downregulating LRG1 in vivo.

Insulin is well recognized as the major activator of de novo lipogenesis (DNL) in the liver (36). However, under obesity and type 2 diabetes conditions, elevated lipogenic capacity persists despite severe insulin resistance (37). While the mechanisms of such paradox are not completely understood, we found that LRG1 treatment, which suppresses insulin signaling, is able to stimulate lipogenesis in hepatocytes. Consistent with this result, LRG1 treatment is sufficient to induce de novo lipogenesis without insu- 
lin presence. Together with the finding that knockout of LRG1 suppressed the activation of SREBP1 but not its expression, these results suggest that LRG1 may promote lipogenesis through an SREBP1-dependent but insulin-independent novel mechanism. This observation would provide an answer to the paradox that lipogenesis is enhanced in the liver despite severe insulin resistance under obesity and type 2 diabetes conditions.

One important question yet to be answered is how LRG1 regulates insulin signaling and lipid metabolism in the liver. As a secretory molecule, LRG1 may regulate liver metabolism by binding to an as-yet-unidentified membrane receptor in hepatocytes. Identification of the LRG1 receptor and/or its downstream targets would thus shed light on the signaling mechanism by which LRG1 inhibits insulin signaling and promotes hepatosteatosis. It has been reported that LRG1 could exert its function by binding to TGFBR2 to modulate TGF- $\beta$ signaling in cancer and endothelial cells $(12,15,38,39)$. However, although dysregulation of TGF- $\beta$ signaling has been implicated in the development of insulin resistance $(40,41)$ and nonalcoholic fatty liver disease (NAFLD; ref. 42), we found that neither knockout of Tgfbr2 nor disrupting TGF- $\beta$ signaling had an effect on LRG1-mediated suppression of insulin signaling in hepatocytes. These data suggest that the action of LRG1 on hepatic insulin signaling and action is independent of TGF- $\beta$ signaling. It is interesting to note that the serum levels of LRG1 are relatively high (about 2.03-50 $\mu \mathrm{g}$ / $\mathrm{mL}$; ref. 11, 43). Given that high levels of serum proteins may function as carries to transport lipids, hormones, vitamins, and minerals in the circulatory system, we cannot exclude the possibility that LRG1 may exert its function by interacting with other serum factors, rather than functioning as a hormone to bind to its membrane receptor. Further studies are needed to elucidate the signaling mechanism of LRG1 action.

While our results show that liver is a major target tissue for LRG1 binding and action, a weak LRG1 binding was also detected in other metabolic tissues such as adipose tissues. Our data show that LRG1 treatment had an inhibitory effect on insulin-stimulated Akt phosphorylation in hepatocytes, but not in adipocytes, revealing a selective effect of LRG1 on liver. This could be due to selective expression of an LRG1 receptor or specific LRG1-associated signaling molecules in hepatocytes. Thus, the improved insulin sensitivity in adipose and muscle tissues of the $\operatorname{Lrg} 1^{\mathrm{KO}}$ mice in vivo is most likely caused by a secondary effect resulting from liver-mediated improvement of whole-body energy homeostasis. In line with this, LRG1 deficiency had only a small effect on the expression of lipolytic and lipid uptake genes in adipose tissue of HFD-fed mice. In addition, no difference in cold stress-induced thermogenic gene expression was detected between WT and $\operatorname{Lrg} 1^{\mathrm{KO}}$ mice. Besides liver, LRG1 binding signals were also detected in bone, pancreas, kidney, and heart, but the role of LRG1 in obesity-induced metabolic dysfunction in these organs remains elusive. Based on our finding that LRG1 is positively associated with insulin resistance as well as other reports linking the role of this protein with diabetic kidney disease (38), inflammation (44), and heart failure (45), it is possible that increased LRG1 binding may contribute to obesity-induced metabolic disturbance in these organs. Further studies will be required to test these possibilities.
In summary, we uncover LRG1 as an adipokine whose expression and secretion are positively correlated with obesity in both humans and mice. We also provide evidence that LRG1 plays a key role in mediating obesity-induced hepatosteatosis and insulin resistance, suggesting that suppressing LRG1 levels and function may be an effective therapeutic treatment for obesityinduced metabolic diseases.

\section{Methods}

Experimental materials. Primer sequences used in this study are listed in Supplemental Table 1. Details of antibodies used in this study are listed in Supplemental Table 2. Sources of cell lines and animals used in this study are listed in Supplemental Table 3. For generation of LRG1 antibody, 3 peptide fragments of mouse LRG1 protein (HGPTEFPSSLPA, RLQRLEDSLLAP, KGQRLLDVAELG) were used for injection to produce homemade rabbit antibody. The specificity of LRG1 antibody was validated in Western blots by comparing $\operatorname{Lrg} 1^{\mathrm{KO}}$ to WT mice tissue samples which showed absence of LRG1 protein bands in serum and adipose tissues of $\operatorname{Lrg} 1^{\mathrm{KO}}$ samples (Supplemental Figure 2B).

Human samples and study approval. Human serum and adipose tissue samples were provided by Christie Bialowas in the Department of Surgery at the University of Texas Health San Antonio (UTHSA) through collaboration (46). Human liver samples were nonpathological tissue obtained from patients undergoing hepatectomy for metastatic disease (such as pancreatic carcinoma/gallbladder carcinoma). Body mass index was calculated as weight divided by squared height value. Serum samples were collected by centrifuging whole blood at $800 \mathrm{~g}$ for 10 minutes at $4^{\circ} \mathrm{C}$. Subcutaneous adipose tissues and liver tissues were isolated and immediately frozen in liquid nitrogen, then transferred into $-80^{\circ} \mathrm{C}$ freezer for long-term storage.

Animal studies. All animal studies were performed in accordance with the guidelines approved by the IACUC of University of Texas Health San Antonio (UTHSA). $\operatorname{Lrg} 1^{\mathrm{KO}}$ mice were obtained from the Knockout Mouse Project (KOMP Repository, UC Davis) in C57BL/6J background. Strategically, genomic sequence of $\operatorname{Lrg} 1$ (which contains 2 exons) was replaced by a targeting cassette (contains a $\beta$-galactosidase gene and selection marker which can be removed in the presence of Cre recombinase). WT and homozygous knockout littermates were acquired by breeding heterozygous to heterozygous mice. All animal experiment groups were randomly assigned with mice of desired genotype. Mice were housed under 12-hour light/dark cycles with free access to food and water. For chow feeding, mice were fed with Teklad laboratory diet (ENVIGO, catalog 7012, with 17\% calories from fat). Cold stress experiments were performed as previously described (47). In brief, mice were housed individually (with free access to food and water) and kept at $4^{\circ} \mathrm{C}$ for 4 hours/day for a total of 4 days; fat tissues were then harvested for further analysis. For HFD feeding, mice were fed with a diet containing $45 \%$ of calories from fat (Research Diets Inc., catalog D12451), starting at 8 weeks of age for 16 weeks. Body weight was measured weekly, body composition was measured using quantitative magnetic resonance imaging (qMRI), and metabolic cage study was performed using Oxymax-CLAMS (Comprehensive Lab Animal Monitoring System) in the Healthspan and Functional Assessment Core of UTHSA. Food intake was measured daily with individual housing. For glucose tolerance test (GTT), mice were prehandled daily for 1 week before overnight fasting under singly housed conditions, blood glucose levels of the mice were measured using glucose meter 
pre- and postinjection of glucose intraperitoneally. For insulin tolerance test (ITT), mice were fasted for 4 hours in the morning before injected with insulin, glucose levels were determined by glucose meter (Bionime) at different time points, and insulin levels were measured using insulin ELISA kit (ALPCO, catalog 80-INSMS-E10).

Adipocyte differentiation and treatment. Brown adipocyte cell lines were maintained in growth medium (DMEM with 10\% FBS and 1\% penicillin-streptomycin). Two days after confluence (day 0), differentiation was induced by adding IBMX $(0.5 \mathrm{mM})$, indomethacin $(125$ $\mu \mathrm{M})$, dexamethasone $(1 \mu \mathrm{M})$, insulin $(20 \mathrm{nM})$, and $\mathrm{T} 3(1 \mathrm{nM})$, and cells were cultured for 3 days. Cells were then maintained in growth medium containing insulin $(20 \mathrm{nM})$ and $\mathrm{T} 3(1 \mathrm{nM})$ until fully differentiated. Cells were incubated with fresh growth medium before further treatments. 3T3-L1 preadipocytes were maintained in growth medium (DMEM with $10 \%$ fetal calf serum and $1 \%$ penicillin-streptomycin). Two days after confluence, differentiation was induced by adding IBMX $(0.5 \mathrm{mM})$, dexamethasone $(1 \mu \mathrm{M})$, and insulin $(1 \mu \mathrm{g} / \mathrm{mL})$ and cells were cultured for 3 days. Cells were then cultured in growth medium containing insulin $(1 \mu \mathrm{g} / \mathrm{mL})$ for 2 more days followed by maintenance in the growth medium. For primary adipocyte studies, adipose tissue SVF cells were isolated according to a procedure previously described (47), and cells were cultured in DMEM containing $20 \%$ FBS and induced for differentiation based on the same procedure for cell lines until fully differentiated.

Identification of secretory proteins from adipocytes. Brown adipocyte cell line and 3T3-L1 cell line were cultured and induced for differentiation according to a protocol previously described (47). Total RNA was isolated from cells before and after differentiation, and gene expression was measured using GeneChip 3' IVT Express Kit (Affymetrix, Inc., catalog P/N901229). Data analysis was performed according to the manufacturer's instructions. Differentially expressed genes were selected using 2-fold difference and an adjusted $P$ value (false positive rate) of less than or equal to 0.05 as the cutoff criteria, and further annotated using MetazSecKB database (http://proteomics.ysu.edu/secretomes/animal/index.php) in order to identify secretory factors.

Lipid content measurement. Serum and tissue levels of triglyceride were measured using a triglyceride colorimetric assay kit (Cayman Chemical, catalog 10010303) according to the manufacturer's instructions. Cholesterol levels were measured using a total cholesterol and cholesteryl ester colorimetric kit (BioVision, catalog K603-100) following the manufacturer's instructions.

Histology. For tissue histology, samples were harvested and fixed in $4 \%$ formaldehyde and embedded in paraffin. Tissue sections ( 5 $\mu \mathrm{m}$ thickness) were prepared and stained with hematoxylin and eosin (H\&E) using standard protocol (48). Oil Red O staining was used to visualize lipid droplets within tissue sections. Briefly, fresh tissues were isolated and prepared into frozen sections ( $5-\mu \mathrm{m}$ thickness) and fixed in 10\% neutral buffered formalin. Sections were incubated with Oil Red O solution and Mayer's Hematoxylin, washed with water, and pictures were taken immediately.

Adipocyte size and number measurement. Adipocyte size was measured on H\&E-stained sections using Image J software. Total adipocyte numbers of each fat pad were determined according to the procedure as described in previous reports $(47,49)$. In brief, mean adipocyte diameter was measured on H\&E sections with Image J software. Adipocyte density (cells/unit volume) was calculated based on adipocyte diameter assuming cubic closest packing. Adipocyte numbers of fat pads were calculated based on fat pad volume (calculated based on weight) and adipocyte density (cells/unit volume).

Primary hepatocyte isolation. Primary hepatocytes were isolated following a procedure previously described (50), with minor modification. In brief, 2- to 4-month-old male mice were anesthetized, liver was first perfused with Hanks' Balanced Saline (HBSS) containing $0.5 \mathrm{mM}$ EGTA and digested with collagenase (Sigma, catalog C-6885; $0.05 \%$ collagenase in HBSS with 1\%BSA). Cells were filtered through 2 layers of gauze, resuspended, and collected through centrifuge before being seeded into collagen-coated plates in William's E medium (Life Technologies, catalog 12551032) supplemented with 5\% FBS and GlutaMax (Gibco, catalog 35050-061).

Glucose output assay. For measuring gluconeogenesis, primary hepatocytes were rinsed with prewarmed PBS and serum starved in glucose-free DMEM medium overnight before medium was replaced with fresh glucose-free DMEM (without phenol red) containing $20 \mathrm{mM}$ sodium lactate and $2 \mathrm{mM}$ sodium pyruvate. For induction of glucose production, $1 \mu \mathrm{M}$ dexamethasone and $500 \mu \mathrm{M}$ 8-bronoadenosine $3^{\prime}$-, $5^{\prime}$-cyclic monophosphate (8-Br-cAMP) were included in the medium. For insulin-induced suppression of glucose production, $10 \mathrm{nM}$ insulin was included in the medium. Condition medium was collected after a 6-hour incubation, and glucose concentration was measured using a colorimetric glucose assay kit (Life Technologies, catalog A22189) following the manufacturer's instructions. Cells were harvested and protein lysate concentration was measured using bicinchoninic acid method and used to normalize glucose production readings.

LRG1 protein expression and purification. LRG1 protein was obtained through our collaboration with Fang Zhang at Novo Nordisk. Briefly, LRG1 was overexpressed in HEK 293 cell line via transfection of pcDNA3.1A-Lrg1-Myc-His plasmid. Culture medium containing LRG1-Myc-His fusion protein was collected and purified using Ni-NTA Agarose. Protein was further purified using ion exchange and size exclusion column. The purity and identity of final product were verified using SEC-HPLC, Coomassie blue staining, and mass spectrometry.

Tissue binding assay. Tissue binding assay was performed according to a procedure previously described $(51,52)$. pCMV-SEAP-Lrg1 plasmid was constructed by inserting mouse Lrg1 cDNA sequence (without the first 96 nucleotides which encode signal peptide) into pCMV-SEAP vector via XbaI restriction enzyme site. PCMV-SEAP and pCMV-SEAP-Lrg1 constructs were then transfected into 293T cells using lipofectamine 2000 reagent. Twenty-four hours after transfection, cells were replaced with serum-free medium and cultured for an additional 48 hours before collection. For in vitro tissue binding assay, thick cut $(40 \mu \mathrm{m})$ frozen tissue slides were prepared from 6-month-old male C57BL/6J mice, and tissue sections were incubated with condition medium containing SEAP or SEAP-LRG1 at room temperature for 1 hour before being washed with PBS. After fixing in acetone-formalin solution (65\% vol/vol acetone, $8 \% \mathrm{vol} / \mathrm{vol}$ formalin, $20 \mathrm{mM}$ HEPES, $\mathrm{pH} 7.0)$, tissue endogenous alkaline phosphatase was inactivated at $65^{\circ} \mathrm{C}$ for 15 minutes before BCIP/NBT substrates were incubated with the sections in order to detect positive binding of SEAP-LRG1 fusion protein. For competitive binding, LRG1-Myc-His fusion protein was transiently expressed in 293T cells, collected in serum-free medium, and used for preincubation with tissue sections before SEAP or SEAPLRG1 condition medium. 
Evaluation of LRG1 target tissue in vivo. Quantitative biodistribution of exogenous LRG1 was determined using fluorescence labeling in combination with NIR imaging (51). Purified LRG1-Myc-His protein was labeled with Vivo Tag 680XL using a NIR Fluorochrome labeling kit (PerkinElmer, catalog NEV11118) according to the manufacturer's instructions. Degree of labeling (DOL) was calculated in order to determine the amount of Tag for control group injection. Labeled protein Tag-LRG1 was injected intravenously into male C57BL/6J mice (4-6 months of age) at $5 \mu \mathrm{g} / \mathrm{g}$ body weight dosage. Control group was injected with equal amount of Tag fluorophore. The distribution of florescence signal was monitored using IVIS Spectrum in vivo imaging system (Optical Imaging Facility at UT Health San Antonio). Both whole-body and tissue florescence signal was recorded, and the radiance (photons/second $/ \mathrm{cm}^{2} /$ steradian) values of each tissue at different time points after injection were used for quantification of Tag or Tag-LRG1 binding.

RNA sequencing and pathway analysis. mRNA was isolated using Trizol method and was further processed for sequencing analysis in the Genomic Sequencing Facility in the Greehey Children's Cancer Research Institute at UT Health San Antonio using next-generation sequencing (NGS) on a HiSeq 3000 system. Gene Ontology (GO) term analysis was performed using the Database for Annotation, Visualization and Integrated Discovery (DAVID) (https://david. ncifcrf.gov/) $(53,54)$ and innateDB (https://www.innatedb.com/) (55), and was also double confirmed using Ingenuity Pathway Analysis (IPA) (Qiagen).

Gene expression analysis. Total RNA from tissues and cells were isolated using TRIzol method. RNA $(1 \mu \mathrm{g})$ from each sample was used for reverse transcription following instructions from QuantiTect Reverse Transcription kit (Qiagen, catalog 205314). For qPCR analysis, gene expression levels were detected using SYBR Green (Applied Biosystems, catalog A25742) method and the reaction was carried out using C1000 Touch Thermal Cycler (Bio-Rad) in the UT Health San Antonio Biobanking and Genome Analysis Core. The relative gene expression was normalized to endogenous housekeeping gene $\beta$-actin levels using $\Delta \Delta \mathrm{CT}$ method, data are presented as fold change over control, unless otherwise indicated.

Immunoblotting analysis. Total protein lysates were prepared by homogenizing tissue in lysis buffer that contains $50 \mathrm{mM}$ HEPES $(\mathrm{pH}$ 7.6), $150 \mathrm{mM} \mathrm{NaCl}, 20 \mathrm{mM}$ Na pyrophosphate, $20 \mathrm{mM} \beta$-glycerophosphate, $10 \mathrm{mM} \mathrm{NaF}, 1 \% \mathrm{NP}-40$, and 10\% glycerol. Proteinase inhibitors (GenDEPOT, catalog P3200-020) were freshly added to the buffer. An equal amount $(20 \mu \mathrm{g})$ of samples was loaded into $8 \%$ to $12 \%$ SDSPAGE gel and resolved by electrophoresis. Proteins were transferred onto nitrocellulose membrane, blocked in $1 \%$ bovine serum albumin, and incubated with primary antibodies at $4^{\circ} \mathrm{C}$ overnight. The blots were then incubated with HRP-conjugated secondary antibody and developed by ECL method.

Lipogenesis assay. Lipogenesis assays were performed according to a previously described procedure (56). In brief, primary hepatocytes were plated onto 12-well plates and cultured overnight in serumfree medium containing PBS, LRG1 $(20 \mu \mathrm{g} / \mathrm{mL})$, insulin $(100 \mathrm{nM})$, or LRG1 plus insulin. Cells were rinsed with PBS and incubated with serum-free medium containing $10 \mu \mathrm{M}$ cold acetate and $0.5 \mu \mathrm{Ci} / \mathrm{mL}$ $\left[1,2-{ }^{14} \mathrm{C}\right]$-acetic acid (PerkinElmer, catalog NEC553050UC) for 2 hours. After washing twice with PBS, the cells were lysed with $0.1 \mathrm{~N}$ $\mathrm{HCl}$. Lipid was extracted using Chloroform-methanol (2:1, vol/vol), lower phase was used for measuring ${ }^{14} \mathrm{C}$ contents. Protein extraction was used to calibrate the results.

Fatty acid oxidation assay. Fatty acid oxidation assays were performed according to a similar procedure as previously described (57). Briefly, primary hepatocytes were cultured in $25 \mathrm{~T}$ flasks overnight with serum-free medium in the presence or absence of LRG1 $(20 \mu \mathrm{g} /$ $\mathrm{mL}$ ). [1-14 C]-Palmitic acid (Moravek-Biochemicals, $53 \mathrm{mCi} / \mathrm{mmol}$ ) was dried under nitrogen gas and resuspended in $\alpha$-cyclodextrin. Cells were rinsed with PBS and incubated with $1 \mathrm{~mL}$ fresh serum-free medium containing $0.417 \mu \mathrm{Ci} /$ flask $\left[1-{ }^{14} \mathrm{C}\right]$-palmitic acid for 30 minutes at $37^{\circ} \mathrm{C}$. Flasks were capped with a rubber stopper with filter paper containing $\mathrm{KOH}$. Reactions were stopped by adding $2.6 \mathrm{~N} \mathrm{HClO}_{4}$ and $\mathrm{CO}_{2}$ was trapped for 2 hours before the filter paper was removed for counting ${ }^{14} \mathrm{C}$ signal. Cells were lysed for protein extraction to calibrate between samples.

Accession number and data sharing. Raw data and processed data of microarray and RNA-Seq in this study were deposited in the NCBI Gene Expression Omnibus database (GEO GSE185484).

Statistics. All data are mean \pm SEM unless specified. For animal experiments, all mice were age-matched and assigned randomly to different treatment groups to avoid potential bias. All results were representative of at least 3 repeated experiments or as indicated. Unpaired 2 -tailed $t$ test was used for the comparison between 2 groups and 1-way ANOVA was used for the comparison of multiple groups. The statistical analysis was performed by using GraphPad prism 8 and Microsoft Excel. $P$ less than or equal to 0.05 was considered statistically significant.

Study approval. All human sample study protocols were approved by either the IRB of the UT Health San Antonio (protocol no. HSC20160323N) or the Second Xiangya Hospital (protocol no. 2020-072).

\section{Author contributions}

SH, JR, JL, HL, JB, PRL, JW, YL, ZS, WX, and FD performed experiments and acquired and analyzed the data. SH wrote the first draft of the manuscript. FZ, BJN, MZ, JLL, RD, and YS contributed to discussion, data analysis, and editing of the text. FL, JB, and LQD contributed to conceptualization and design, data analysis and interpretation, manuscript writing, and financial support of the study.

\section{Acknowledgments}

This work was supported in part by grants from the NIH (DK102965), the American Diabetes Association (1-19-IBS-147), the National Key Research and Development Program of China (2019YFA0801903 and 2018YFC2000100), the National Natural Science Foundation of China (81730022), and the Baptist Health Foundation of San Antonio (2020 Strategic to Mission). We are thankful for the technical support from the Biobanking and Genome Analysis Core, the Core Optical Imaging Facility (supported by UTHSA and NIH-NCI P30 CA54174), the Genome Sequencing Facility (supported by UTHSA, NIH-NCI P30 CA054174, NIH Shared Instrument grant 1S10OD021805-01, and CPRIT Core Facility Award RP160732), and the Nathan Shock Center Aging Animal Models and Longevity Assessment Core (supported by the Nathan Shock Center for Excellence in Basic Biology of Aging grant AG13319), and the Integrated Physiology of Aging Core. We are also thankful for assistance from Novo 
Nordisk for protein purification and mass spectrometry analysis. We thank Luzhe Sun (UTHSA) for Huh7 control and sh-smad4 cell lines and Christie Bialowas (UTHSA) for providing human serum and adipose samples.
Address correspondence to: Lily Q. Dong or Juli Bai, 7703 Floyd Curl Drive, San Antonio, Texas 78229, USA. Phone: 210.567.4849; Email: dongq@uthscsa.edu (LQD); Phone: 210.567.4526; Email: baij@uthscsa.edu (JB).
1. Kwon H, Pessin JE. Adipokines mediate inflammation and insulin resistance. Front Endocrinol (Lausanne). 2013;4:71.

2. Rasouli N, Kern PA. Adipocytokines and the metabolic complications of obesity. JClin Endocrinol Metab. 2008;93(11 suppl 1):S64-S73.

3. Kim S, Moustaid-Moussa N. Secretory, endocrine and autocrine/paracrine function of the adipocyte. J Nutr. 2000;130(12):3110S-3115S.

4. Rabe K, et al. Adipokines and insulin resistance. Mol Med. 2008;14(11-12):741-751.

5 . Ouchi N, et al. Adipokines in inflammation and metabolic disease. Nat Rev Immunol. 2011;11(2):85-97.

6. $\mathrm{Mu} \mathrm{H}$, et al. Adipokine resistin promotes in vitro angiogenesis of human endothelial cells. Cardiovasc Res. 2006;70(1):146-157.

7. Minokoshi Y, et al. Leptin stimulates fatty-acid oxidation by activating AMP-activated protein kinase. Nature. 2002;415(6869):339-343.

8. Maeda N, et al. Diet-induced insulin resistance in mice lacking adiponectin/ACRP30. Nat Med. 2002;8(7):731-737.

9. Yang $Q$, et al. Serum retinol binding protein 4 contributes to insulin resistance in obesity and type 2 diabetes. Nature. 2005;436(7049):356-362.

10. Jung UJ, Choi MS. Obesity and its metabolic complications: the role of adipokines and the relationship between obesity, inflammation, insulin resistance, dyslipidemia and nonalcoholic fatty liver disease. Int J Mol Sci. 2014;15(4):6184-6223.

11. Haupt H, Baudner S. [Isolation and characterization of an unknown, leucine-rich 3.1-S-alpha2-glycoprotein from human serum (author's transl)]. Hoppe Seylers Z Physiol Chem. 1977;358(6):639-646.

12. Wang $X$, et al. LRG1 promotes angiogenesis by modulating endothelial TGF- $\beta$ signalling. Nature. 2013;499(7458):306-311.

13. Wang CH, et al. LRG1 expression indicates unfavorable clinical outcome in hepatocellular carcinoma. Oncotarget. 2015;6(39):42118-42129.

14. Zhong D, et al. LRG1 modulates invasion and migration of glioma cell lines through TGF- $\beta$ signaling pathway. Acta Histochem. 2015;117(6):551-558.

15. Zhou Y, et al. LRG1 promotes proliferation and inhibits apoptosis in colorectal cancer cells via RUNX1 activation. PLoS One. 2017;12(4):e0175122.

16. Pek SL, et al. Elevation of a novel angiogenic factor, leucine-rich- $\alpha 2$-glycoprotein (LRG1), is associated with arterial stiffness, endothelial dysfunction, and peripheral arterial disease in patients with type 2 diabetes. J Clin Endocrinol Metab. 2015;100(4):1586-1593.

17. Watson CJ, et al. Proteomic analysis of coronary sinus serum reveals leucine-rich $\alpha 2$-glycoprotein as a novel biomarker of ventricular dysfunction and heart failure. Circ Heart Fail. 2011;4(2):188-197.

18. Nakajima M, et al. Brain localization of leucine- rich $\alpha 2$-glycoprotein and its role. Acta Neurochir Suppl. 2012;113:97-101.

19. Fujimoto M, et al. Leucine-rich $\alpha 2$-glycoprotein as a potential biomarker for joint inflammation during anti-interleukin-6 biologic therapy in rheumatoid arthritis. Arthritis Rheumatol. 2015;67(8):2056-2060.

20. Sarvari AK, et al. Plasticity of epididymal adipose tissue in response to diet-induced obesity at single-nucleus resolution. Cell Metab. 2021;33(2):437-453.

21. Gesta S, et al. Evidence for a role of developmental genes in the origin of obesity and body fat distribution. Proc Natl Acad Sci U S A. 2006;103(17):6676-6681.

22. Naka T, Fujimoto M. LRG is a novel inflammatory marker clinically useful for the evaluation of disease activity in rheumatoid arthritis and inflammatory bowel disease. Immunol Med. 2018;41(2):62-67.

23. Titchenell PM, et al. Hepatic insulin signalling is dispensable for suppression of glucose output by insulin in vivo. Nat Commun. 2015;6:7078.

24. Korbonits M, et al. Metabolic and hormonal changes during the refeeding period of prolonged fasting. Eur J Endocrinol. 2007;157(2):157-166.

25. O'Donnell LC, et al. Molecular characterization and expression analysis of leucine-rich alpha2-glycoprotein, a novel marker of granulocytic differentiation. JLeukoc Biol. 2002;72(3):478-485.

26. Xie ZB, et al. LRG-1 promotes pancreatic cancer growth and metastasis via modulation of the EGFR/p38 signaling. J Exp Clin Cancer Res. 2019;38(1):75.

27. Sandanayake NS, et al. A combination of serum leucine-rich alpha-2-glycoprotein 1, CA19-9 and interleukin- 6 differentiate biliary tract cancer from benign biliary strictures. Br J Cancer. 2011;105(9):1370-1378.

28. Shirai R, et al. Up-regulation of the expression of leucine-rich alpha(2)-glycoprotein in hepatocytes by the mediators of acute-phase response. Biochem Biophys Res Commun. 2009;382(4):776-779.

29. Ono K, et al. Identification of microRNA that represses IRS-1 expression in liver. PLoS One. 2018;13(1):e0191553.

30. Kerouz NJ, et al. Differential regulation of insulin receptor substrates-1 and -2 (IRS-1 and IRS-2) and phosphatidylinositol 3-kinase isoforms in liver and muscle of the obese diabetic (ob/ob) mouse. J Clin Invest. 1997;100(12):3164-3172.

31. Kovacs P, et al. The role of insulin receptor substrate-1 gene (IRS1) in type 2 diabetes in Pima Indians. Diabetes. 2003;52(12):3005-3009.

32. Besse-Patin A, et al. PGC1A regulates the IRS1:IRS2 ratio during fasting to influence hepatic metabolism downstream of insulin. Proc Natl Acad Sci U S A. 2019;116(10):4285-4290.

33. Ide T, et al. SREBPs suppress IRS-2-mediated insulin signalling in the liver. Nat Cell Biol.
2004;6(4):351-357.

34. Jhala US, et al. cAMP promotes pancreatic betacell survival via CREB-mediated induction of IRS2. Genes Dev. 2003;17(13):1575-1580.

35 . Rice $\mathrm{S}$, et al. Action of metformin on the insulinsignaling pathway and on glucose transport in human granulosa cells. J Clin Endocrinol Metab. 2011;96(3):E427-E435.

36. Ferre P, Foufelle F. Hepatic steatosis: a role for de novo lipogenesis and the transcription factor SREBP-1c. Diabetes Obes Metab. 2010;12(suppl 2):83-92.

37. Brown MS, Goldstein JL. Selective versus total insulin resistance: a pathogenic paradox. Cell Metab. 2008;7(2):95-96.

38. Hong Q, et al. LRG1 promotes diabetic kidney disease progression by enhancing TGF$\beta$-induced angiogenesis. J Am Soc Nephrol. 2019;30(4):546-562.

39. Meng $\mathrm{H}$, et al. LRG1 promotes angiogenesis through upregulating the TGF- $\beta 1$ pathway in ischemic rat brain. Mol Med Rep. 2016;14(6):5535-5543.

40. Hong SH, et al. High fat diet-induced TGF- $\beta / G b b$ signaling provokes insulin resistance through the tribbles expression. Sci Rep. 2016;6:30265.

41. Yadav $\mathrm{H}$, et al. Protection from obesity and diabetes by blockade of TGF- $\beta / \operatorname{Smad} 3$ signaling. Cell Metab. 2011;14(1):67-79.

42. Yang $\mathrm{L}$, et al. Transforming growth factor beta signaling in hepatocytes participates in steatohepatitis through regulation of cell death and lipid metabolism in mice. Hepatology. 2014;59(2):483-495.

43. Weivoda S, et al. ELISA for human serum leucinerich alpha-2-glycoprotein-1 employing cytochrome $\mathrm{c}$ as the capturing ligand. JImmunol Methods. 2008;336(1):22-29.

44. Pek SLT, et al. Association of circulating proinflammatory marker, leucine-rich- $\alpha 2$-glycoprotein (LRG1), following metabolic/bariatric surgery. Diabetes Metab Res Rev. 2018;34(7):e3029.

45. Song W, Wang X. The role of TGF $\beta 1$ and LRG1 in cardiac remodelling and heart failure. Biophys Rev. 2015;7(1):91-104.

46. Ryu J, et al. Potential roles of adiponectin isoforms in human obesity with delayed wound healing. Cells. 2019;8(10):1134.

47. Liu M, et al. Grb10 promotes lipolysis and thermogenesis by phosphorylation-dependent feedback inhibition of mTORC1. Cell Metab. 2014;19(6):967-980.

48. Fischer AH, et al. Hematoxylin and eosin staining of tissue and cell sections. CSH Protoc. 2008;2008:pdb prot4986.

49. Mancuso DJ, et al. Genetic ablation of calciumindependent phospholipase A2gamma prevents obesity and insulin resistance during high fat feeding by mitochondrial uncoupling and increased adipocyte fatty acid oxidation. J Biol Chem. 2010;285(47):36495-36510.

50. Galan-Davila AK, et al. Alternative splicing 
variant of the scaffold protein APPL1 suppresses hepatic adiponectin signaling and function. J Biol Chem. 2018;293(16):6064-6074.

51. Ntziachristos V, et al. Fluorescence imaging with near-infrared light: new technological advances that enable in vivo molecular imaging. Eur Radiol. 2003;13(1):195-208.

52. Li E, et al. OLFR734 mediates glucose metabolism as a receptor of asprosin. Cell Metab. 2019;30(2):319-328.
53. Huang da W, et al. Systematic and integrative analysis of large gene lists using DAVID bioinformatics resources. Nat Protoc. 2009; 4(1):44-57.

54. Huang da W, et al. Bioinformatics enrichment tools: paths toward the comprehensive functional analysis of large gene lists. Nucleic Acids Res. 2009; 37(1):1-13.

55. Breuer K, et al. InnateDB: systems biology of innate immunity and beyond--recent updates and continuing curation. Nucleic Acids Res.
2012;41(d1):D1228-D1233.

56. Akie TE, Cooper MP. Determination of fatty acid oxidation and lipogenesis in mouse primary hepatocytes. JVis Exp. 2015;102(102):e52982.

57. Antinozzi PA, et al. Molecular or pharmacologic perturbation of the link between glucose and lipid metabolism is without effect on glucose-stimulated insulin secretion. A re-evaluation of the long-chain acyl-CoA hypothesis. J Biol Chem.1998; 273(26):16146-16154. 